Meeting report

\title{
The $\mathbf{2 6}^{\text {th }}$ Congress of the Scandinavian Society of Anaesthesiology and Intensive Care Medicine, Tromso, Norway, 13-17 June 2001
}

Investigational Drugs database

http://www.iddb3.com, Current Drugs Ltd, London, UK

Correspondence: Investigational Drugs database, info@current-drugs.com

Published online: 13 July 2001

Critical Care 2001, 5:204-206

(c) 2001 Current Drugs Ltd (Print ISSN 1364-8535; Online ISSN 1466-609X)

\begin{abstract}
The $26^{\text {th }}$ Congress of the Scandinavian Society of Anaesthesiology and Intensive Care Medicine took place in the state-of-the art Tromso University Hospital. There were over 500 participants, and approximately 300 oral and poster presentations highlighted the latest progress in diverse areas. Much interest focused on activated protein $C$ (APC) and other ways forward in sepsis treatment, pain management, novel markers of neurotrauma and antioxidants in bypass surgery. The meeting continues to be the leading anaesthesiology and intensive care conference in the region.
\end{abstract}

Keywords anesthesiology, congresses, intensive care, pain, sepsis

The Congress took place at the northernmost academic center in the world, in the state-of-the art Tromso University Hospital. There were over 500 participants, mainly from Scandinavian countries, with approximately 50 invited participants from non-Scandinavian countries, primarily from Europe and the USA. The meeting was managed and organized by a local committee, chaired by the University of Tromso Professor of Anaesthesiology Dr Lars Bjertneas. Approximately 300 oral and poster presentations highlighted the latest progress in diverse areas of anaesthesiology and intensive care medicine from Scandinavia. Invited speakers held state-of-the art lectures in selected areas of pathophysiology and therapy. The meeting benefited from the support of various industry sponsors, and the abstracts of the meeting were published in a supplement of Acta Anesthesiologica Scandinavica.

\section{Sepsis: the PROWESS study}

Sepsis represents a major cause of mortality, affecting 750,000 patients/year in the USA, with 2000 new cases identified each day and 700 deaths per year (2001 US statistics). Much interest focused on the haemostatic aspects of sepsis and septic shock. This interest stems partly from the recent results of a multicenter trial that demonstrated the beneficial effects and a significant survival benefit provided by APC infusion in septic patients. Dr P Laterre (University Clinic, St Luc, Belgium) provided a detailed overview of the recently completed clinical trial with APC (or Drotecogin alpha; $24 \mu \mathrm{g} / \mathrm{kg}$ per $\mathrm{h}$ for 4 days by intravenous infusion). In the PROWESS (Protein C Worldwide Evaluation in Severe Sepsis) study, a 1690patient randomized trial that utilized inclusion criteria similar to those of the preceding anticytokine trials, the mortality rate in the placebo group was 30.8\%, which decreased to $24.9 \%$ in the treatment group. This corresponds to a $6 \%$ reduction in mortality, which means that for every 16 patients treated with APC one can expect to save an additional life.

That trial demonstrated no subgroup differences in outcome, except for the very mild shock group (Acute Physiology and Chronic Health Evaluation II score <19), in which the drug tended to worsen the outcome. No sex, race or age differences were noted in the efficacy of the 
drug. Both the patients who presented with low pretrial APC levels and the patients with normal APC levels benefited, indicating that there is no need for measurements of APC before instituting therapy. Pneumonia and lung infection were common causes of death, because of the fact that during the trial two major influenza epidemics were prevalent throughout the study centers. APC treatment induced a faster decline in plasma IL-6 levels, indicating a possible anti-inflammatory effect of the treatment.

In the APC arm, duration of intensive care unit stay was slightly but significantly reduced, and there was a reduction in catechol requirements and number of days on a ventilator. The trial did not exclude any patients except those with a tendency for higher bleeding complications and severely moribund patients who were expected to die within $24 \mathrm{~h}$. The only noted side effect was related to serious bleeding, of which the incidence increased from 2 to $3.5 \%(P=0.06)$. However, after stopping the APC infusion, the effect of the drug quickly diminished, and no mortality due to bleeding was noted. After many unsuccessful trials, it appears that the first 'magic bullet' has arrived.

\section{Other ways forward in sepsis}

Nevertheless, if we are to improve survival further in sepsis, then there is no doubt that additional therapies are needed, as overviewed by Dr Else Tonnesen (Department of Anesthesiology, Aarhus, Denmark). Potential ways to affect the complement system in the future include the complement regulator $\mathrm{C} 1$ inhibitor, the soluble complement receptor 1 and antibodies that neutralize C5, as overviewed by $\mathrm{Dr}$ Tom Mollnes (Department of Immunology, Nortland Center Hospital, Bodo, Norway). Dr Daniel Traber (Department of Anesthesiology, University of Texas, USA) demonstrated beneficial effects with recombinant antithrombin III in a sepsis/smoke inhalation induced model of severe systemic inflammatory response syndrome in sheep,

Although the Glaxo-Wellcome trial with the nitric oxide (NO) synthase inhibitor $N^{G}$-monomethyl-L-arginine was discontinued some years ago because of lack of survival efficacy, much interest still focuses on the role of NO and related species in the pathogenesis of sepsis. Dr M Kirov (State University Arhangelsk, Russia) and Lars Bjertnaes (University of Tromso, Norway) presented clinical data with methylene blue, a guanylate cyclase inhibitor that presumably acts via blocking NO-induced increases in cGMP levels in the vasculature of septic patients. Administration of methylene blue in 20 patients induced an increase in MAP, and a stabilization of stroke volume and left ventricular stroke index. It reduced the requirement for catecholamines, but did not improve oxygen delivery and did not affect serum chemistry parameters of organ injury.
Dr Salvador Moncada (Wolfson Institute, London, UK) presented novel data on the differential time-dependent and dose-dependent regulation of mitochondrial respiration and cell death by NO. Dr Csaba Szabo (Inotek Corporation, Boston, MA, USA) presented evidence for the pathogenetic role of peroxynitrite, a toxic reaction product of $\mathrm{NO}$, and the protective effect of peroxynitrite decomposition catalysts in various rodent models of shock. Dr Daniel Traber (University of Texas, USA) presented data with the inducible NO synthase inhibitor mercaptoethylguanidine and with the NO scavenging compound pyridoxalated haemoglobin polyoxyethylene in various ovine models of sepsis. Dr Mikael Bodelsson and colleagues (University Hospital Lund, Sweden) presented evidence that the endotoxin-binding antibiotic bactericidal/permeability increasing protein is able to attenuate the expression of inducible NO synthase in septic blood vessels.

In addition to inhibition of $\mathrm{NO}$ and its toxic byproducts, additional interest has emerged in the use of inhaled NO in sepsis as a means to attenuate or reverse some of the pulmonary pathophysiology associated with sepsis. Dr Claes Frostell (Karolinska Institute, Stockholm, Sweden) overviewed this area of research. He concluded that inhaled NO in sepsis appears to improve pulmonary haemodynamics acutely and reduces neutrophil infiltration into the lung, but the long-term benefit in terms of patient survival rate is unclear. Dr Lars Bjertnaes (Department of Anaesthesiology, University of Tromso, Norway) and his group presented data from studies with DS-1, a novel selective pulmonary vasodilator compound, in endotoxaemic sheep. The compound attenuated pulmonary hypertension without affecting systemic haemodynamic parameters, and improved pulmonary extravasation and oxygen extraction. It was speculated that compounds such as this may eventually become a viable future alternative to $\mathrm{NO}$ gas inhalation in the clinical setting.

The proinflammatory side of sepsis and septic shock received less attention at the meeting than in previous meetings, probably due to the disappointments with clinical trials with anticytokine therapies. Dr Helge Opdahl (Ulleval Hospital, Oslo, Norway) overviewed the evidence that shows that traditionally administered catecholamines, in addition to acting as pressor agents, exert effects as immuno/inflammatory modulators. A clear negative correlation between lipopolysaccharide-stimulated $\mathrm{IL}-1$ production and plasma dopamine levels was noted in ex vivo whole-blood assays, supporting the anti-inflammatory effects of catecholamines, presumably acting via cAMP-mediated processes in the mononuclear cells of septic patients. Dr Szabo presented a state-of-the-art lecture on the activation and possible role of nuclear factor- $\kappa B$ activation in sepsis. This factor appears to be a clinical target, because it is clearly elevated in septic 
patients, its levels correlate with the severity of the disease, and inhibition of its activation exerts multiple protective effects in experimental models. The commonly used antioxidant compounds exert nonspecific effects, however, and no preclinical or clinical trials are available with more specific approaches, such as selective inhibitors of I- $\mathrm{KB}$ kinases. Dr Vladimir Kuklin (State University of Arhangelsk, Russia) presented a 100-patient study of severe sepsis, and demonstrated that plasmapheresis reduces mortality rate from 56 to $34 \%$ $(P<0.05)$, presumably by removing toxic metabolic byproducts or proinflammatory mediators in sepsis. Apart from transient episodes of hypotension, no side effects of the treatment were noted.

\section{Pain management}

Pain management was another area of intense interest. Dr Tor Tennessen (Department of Anesthesiology, The National Hospital, Norway) overviewed the new options for treatment and concluded that the following are worthy of pursuing: diminished use of strong opioids; effective doses of paracetamol and nonsteroidal anti-inflammatory drugs; glucocorticoids; and combinations of various nonopioid analgesics. Dr Reijo Korpela (Hospital for Children and Adolescents, Helsinki, Finland) concluded that the combined use of paracetamol and one nonsteroidal antiinflammatory drug would be the optimal method for relieving postoperative pain in children.

\section{Neurotrauma: novel markers and sedation supplements}

Dr Bertil Romner (Department of Neurosurgery, University of Tromso) presented novel data for potential serum markers of traumatic brain injury: neurone-specific enolase, gliar fibrillary acidic protein and S-199 $\beta$ protein. Promising clinical data were presented, especially for the latter protein.

In an intervention study conducted in 427 patients with head trauma, Dr N Juul (Aarhus University Hospital, Denmark) failed to report any added benefit with NMBA (the neuromuscular blocking agent Selfotel) as a supplement to sedation, and questioned the use of such agents as routine part of the sedation strategy.

\section{Antioxidants in recovery from bypass surgery}

In a small-scale study that involved cardiopulmonary bypass patients, a mixture of antioxidants (Vitamins $E$ and $\mathrm{C}$, allopurinol and acetylcysteine) improved endothelial function after surgery. However, further work is needed to determine whether this change will transfer into improved clinical outcomes.

\section{Conclusion}

The meeting continues to be the leading anaesthesiology and intensive care conference in the region. 